\title{
Using vignettes in educational research: a framework for vignette construction
}

\section{Introduction}

In research contexts vignettes are primarily associated with the idea of descriptive episodes simulating real events that are presented either in a written or visual form. In some instances the term 'vignette' is used interchangeably to include terms such as scenarios, stories, case studies, or other variations (Bradbury-Jones et al., 2014; Hughes \& Huby, 2004; Jeffries \& Maeder, 2005; Richman \& Mercer, 2002; Veal, 2002). Some researchers, however, stress the importance of defining vignettes clearly, arguing that such distinctions are central to the research aims (Jeffries \& Maeder, 2005). For example, where the orientation and purpose of the research itself is based upon vignettes, defining and justifying the use of vignettes as the appropriate methodological tool is viewed as paramount (Hughes \& Huby, 2004). Importantly, vignette methodology offers ways to connect research with practice particularly with the potential to bring about transformative experiences by practitionerresearchers (Nind \& Pepin, 2009).

Torres (2009) noted that over the last fifty years or so changes in vignette methodology have taken place, mainly due to changing epistemological and ontological perspectives that "legitimized the usage of qualitative methods in the social sciences" (p.101) and the kind of research questions they are appropriate for addressing. The quality and credibility of qualitative inquiry is strengthened through establishing methodological awareness and consistency (Corbin \& Strauss, 2008; Yilmaz, 2013) where alignment between the theoretical paradigms, epistemological assumptions, research strategies, and choice of methods are emphasized. In this paper, we propose that not only should the choice of methods align with the research design and theoretical paradigms but the methods themselves, in this case vignettes, should also be developed so that they are consistent with the research paradigm. 
Vignettes are used for diverse research purposes, sometimes as the sole method or in conjunction with other methods such as in accompanying quantitative inquiry (Simon \& Tierney, 2011). They are also versatile as a research tool in terms of their style, the way they are used and their applicability to various contexts. Previous research has identified many important elements regarding vignettes through establishing vignette taxonomies (Richman \& Mercer, 2002), comparisons of advantages and disadvantages (Poulo, 2001; Simon \& Tierney, 2011; Torres, 2009), and criteria and guidelines for vignettes construction and use (Bradbury-Jones et al, 2014; Hughes \& Huby, 2004; Jeffries \& Maeder, 2005; Paddam et al., 2010; Simon \& Tierney, 2011). Although vignette studies often discuss the practical and procedural elements of using vignettes, not all explain the relationship between the substance of the vignette material and the underlying theoretical frameworks of the phenomena being investigated and the research paradigm.

The aim of this paper is to investigate the use of purposefully constructed vignettes for gathering data in educational research, especially for eliciting value-laden constructs such as teacher beliefs and understandings and how these influence their practices. Responding to calls for more rigorous criteria for vignette development and implementation (Jeffries \& Maeder, 2005), we aim to contribute to international discourse by proposing a unifying vignette framework to guide the construction of vignettes so they are methodologically consistent with the aims and purpose of the research. We then draw on a study from mathematics education to illustrate how the framework can be used for constructing vignettes. Before discussing elements of the framework and the exemplar study, we discuss (1) different definitions of the notion of 'vignettes' in the literature and specify our own use of the term in this paper and (2) methodological aspects concerning alignment and consistency.

\section{Vignette definitions}


In research contexts vignettes are descriptive episodes of specific situations that simulate real events or problems that are usually presented in written or visual formats. The episodes might be about people, situations, or events. A common purpose for using vignettes is to elicit information through inviting responses, encouraging discussions, and probing for understandings to gain insights to participants' beliefs, emotions, judgments, attitudes and values about the particular phenomenon that lies at the heart of the research. Vignettes are also seen as advantageous for collecting participants' complex thoughts about sensitive or ethical topics (Simon \& Tierney, 2011) and suitable for studying value-laden understandings (Torres, 2009).

Some vignette definitions are broad and overarching. For example, Hughes and Huby (2004) described vignettes as a form of effective stimuli, using text or images to which participants are invited to respond. Jeffries and Maeder (2005) proposed vignettes as “incomplete short stories that are written to reflect, in a less complex way, real-life situations in order to encourage discussions and potential solutions to problems where multiple solutions are possible” (p. 20). Torres (2009) suggested that vignettes depict “specific situations and the problems that might arise in them in order to probe informants about the way they understand these” (p. 94).

In this paper, we draw upon the definitions of Hughes and Huby (2004), Jeffries and Maeder (2005), and Torres (2009) to propose a working definition of vignettes for use in research settings. Specifically, in this paper we consider vignettes to be written, visual, or oral stimuli, aligned with relevant research paradigms and methodologies, reflecting realistic and identifiable settings that resonate with participants for the purpose of provoking responses, including but not limited to beliefs, perceptions emotions, affective responses, reflections, and decision making. 
The qualitative paradigm is interested in understanding a phenomenon from the perspective of the participants involved and reflects a wide variety of theoretical paradigms, methodologies and methods (Yilmaz, 2013). The nature of the research determines the specifics of the paradigms, methodologies and methods. For example, studies seeking to investigate teacher beliefs and understandings reside in the interpretative paradigm and assume a subjective epistemological position. This position views reality as subjective and mediated by the individuals' senses, offering a way to explain differences in value-laden constructs relative to an individual's beliefs, perceptions, experiences, cultures, ideologies and norms (Baghramian \& Carter, 2018). The epistemological assumption of how knowledge can be created, acquired and communicated, proposes that meaning is constructed and understood from the perspective of the individual, and is evidenced in capturing what they believe and the meaning they ascribe to a particular phenomenon.

In seeking to investigate teacher beliefs and understandings, the paradigm and epistemological assumptions act to steer the frameworks, the methodological approach and the choice of suitable methods so that an overall alignment between and within the research design is achieved. In keeping with seeking teacher beliefs and understandings about certain phenomena relevant to teaching and learning, it is appropriate to use methodologies and methods that are situated in the cultural and social settings within classrooms (Cohen, Manion \& Morrison, 2011). In classroom settings where emotional, social and cultural factors are influential, seeking to investigate teachers' beliefs and understandings through the use of vignettes provides for individual teacher subjective views and allows the researcher to reflect on participants' interpretations and to note elements of similarity/difference between them (Stecher et al., 2006). If vignettes are used in conjunction with other data collection methods such as interviews (Simon \& Tierney, 2011), they offer an additional advantage of obtaining other information from participants. This may act to confirm the reliability of, and 
augment information gathered from traditional methods (Jeffries \& Maeder, 2005). Further, thoughtful preparation of the questions accompanying vignettes provides opportunities to sensitively extract information about the phenomenon being investigated. This is particularly important in situations that may be sensitive or ethically difficult, for example when seeking information involving children or vulnerable people.

In the next section our attention turns to the construction of the vignettes. Drawing on a range of vignette research we identify important aspects for vignette construction and propose a vignette framework with three key elements: conception, design, and administration. We suggest that adherence to these key elements when using vignette methods supports aligning the research aims and methodology and serves internal validity.

\section{The Vignette Framework}

Although there are no prevailing processes for constructing vignettes, the formulation of the vignette needs to fit conceptually with the research aims and therefore necessitates consideration of who will be responding to it. Vignette construction appears to be an intricate and iterative process, simultaneously looking outwards to conceptualize the research aims and inwards at the details of construction.

To develop a framework, we recorded aspects and processes from educational research studies where vignettes were used to elicit value-laden constructs. Elements that were seen as belonging together led to the emergence of the tentative core elements and supporting characteristics. Saturation was reached when no additional elements or characteristics emerged. Then a draft framework organizing the core elements and characteristics was formed accompanied by descriptors. 
In Table 1 we present the vignette framework we propose, which organizes three key elements (column 1) that detail twelve characteristics (column 2) with accompanying descriptors (column 3). Next we discuss separately each of the three key elements of the framework (conception, design, and administration) and elaborate on their characteristics.

\section{[Table 1 to be inserted here]}

\section{Conception}

The key element of 'conception', as presented in Table 1, includes characteristics that are central to the research phenomena and the aims of the research, including: capturing content, realistic and hypothetical portrayals, and purpose/function.

Capturing content. An important decision when using vignettes as a research tool is whether to use existing information or to construct new material that captures relevant content that is meaningful to the participants (Cohen, Manion \& Morrison, 2011). Some researchers have used real-life events that are then turned into a story (Bradbury-Jones et al., 2014; Jeffries \& Maeder, 2005; Poulou, 2001). Others draw on existing literature and conceptual frameworks (Simon \& Tierney, 2017), personal or professional experiences (Veal, 2002), practical experiences from the field (Paddam et al., 2010; Stecher et al., 2006), historical case study notes, (Wilson \& While, 1998), or combinations of the aforementioned sources (Simon and Tierney, 2011; Torres, 2009;) to capture vignette content.

For example, in educational research studies, Veal (2002) developed science vignettes based on his personal experience while teaching science to students, where the pedagogical aspects were developed from his teaching practices and corroborated by other science educators. The resulting vignettes were seen to be an effective tool for purposeful interventions by eliciting discussion, promoting problem solving and reflection when researching teacher practices. Stecher et al. (2006) asked a panel of expert mathematicians 
and mathematics educators, in conjunction with examination of national curriculum documents, to distill a taxonomy of reform-orientated curriculum and instructional practices that were reflected in four classroom 'events' that formed the basis of their vignettes. Jeffries and Maeder (2005) used vignettes from a teacher development course to assess teachers’ beliefs and pedagogical understandings and found that vignettes were not only a highly effective assessment tool, but provided "a rich learning experience for eliciting and promoting teacher understanding” (p.18) in a professional learning capacity. In these examples, the classroom context and associated pedagogical practices were an important aspect of the vignette content from which to draw teachers' beliefs and understandings (Baghramian \& Carter, 2018), which in turn were effective for informing practices and sharing understandings between teachers.

Realistic and hypothetical portrayals. Closely linked to capturing vignette content is how they are portrayed to potential participants. It is suggested that, on the one hand, vignette information be concrete enough to approximate the reality of a situation but, on the other hand, be abstract enough to allow participants to form their own interpretations, understandings and beliefs “on topics very familiar to them” (Poulou, 2001, p. 58). Unlike made up situations, vignettes are frequently based on events, episodes, characters, and descriptions of real-life situations.

This distinction is viewed as extremely important because it is this grounding in reality that renders vignettes as being plausible and credible, while also providing some ‘distance’ from actual events. For example, Jenkins et al. (2010) argued that, as vignette narratives unfold, plausibility is crucial and "must be carefully managed with regards to both the participants' emotional state and the validity of data” (p. 30). Bradbury-Jones et al. (2014) discussed the value of vignettes for providing some distance between the researcher and participants particularly when sensitive topics are the subject of vignettes. The distance from 
reality can facilitate the disclosure of information about sensitive issues and also provides protection for the participants from getting upset and embarrassed (Bradbury-Jones et al., 2014; Paddam et al., 2010).

Vignettes are an effective tool for exploring issues or phenomena that is "not easy either for investigators to ask or for respondents to answer” (Stravakou \& Lozgka, 2018, p. 1191) yet they allow participants a level of freedom and power in the research process because their understandings can be unraveled and expressed freely. It may also be the case, as suggested by Hughes and Huby (2004), that where participants are interested in and are actively involved in the research, this may facilitate detailed responses thereby improving the reliability and validity of data. This reinforces the importance of portraying vignette information in ways that resonate with participants so they are able to respond in ways that are effective for capturing their views about the research phenomena.

Particular to educational research, Stecher et al. (2006) suggested that vignettes are more realistic for prompting teacher accounts of instructional practice when they provided a “classroom context in which to situate their responses” (p. 102), but also caution that using vignettes as prompts to elicit teacher responses about their practices does not necessarily reflect their instructional behaviour in classrooms. However, while both the aspect of plausibility and distance are considered especially significant when stimulating responses from research participants, Torres (2009) reminds us of the limitation of vignettes as only representing a "version of reality” (p. 105) and not for inferring potential behaviour. This is because some participants might react differently to the vignette than to actual events due to unanticipated occurrences (Paddam et al., 2010). In any case, being able to probe for participants' beliefs and understandings using vignettes may not necessarily aim to establish accurate predictions of behaviour, but they are still valuable for providing "insight into the 
social components of the participants' interpretive frameworks and perceptual processes” (Jenkins et al., 2010, p. 6).

Purpose/function. As we mentioned earlier, common purposes for using vignettes are to elicit responses, encourage discussions, and probe for understandings. In educational research, Jeffries and Maeder (2005) used vignettes to assess teachers’ pedagogical understandings and found that the vignettes were not only a highly effective assessment tool they also provided “a rich learning experience for eliciting and promoting teacher understanding” (p.18). Other studies have used vignettes to investigate teacher perceptions and attributions relevant to aspects of students' emotional and behavioral difficulties (Poulou, 2001) or to investigate issues of educational assessment (Simon \& Tierney, 2011). Torres (2009) suggested vignettes are helpful for tapping into participants’ beliefs and understandings, by providing a concrete context around a situation so that various factors can be discussed or perspectives unpacked and elaborated on. Specific to mathematics education, Stecher et al. (2006) used vignettes to evoke the complexity of classroom teaching situations and asked teachers to indicate their approaches to mathematics instruction, finding that this method captured important information about reform-orientated practices that survey, logs, and classroom observations did not.

Vignettes may be used as the sole method for eliciting data or in combination with other data collection tools to augment information gathered from more traditional methods (Jeffries \& Maeder, 2005). In several cases vignettes were the sole source of data collection: as prompts for discussion (Poulou, 2001; Torres, 2009); as a stimulus for participants to make individual comments (Beilstein et al., 2017); to provide contrasting situations (Stravakou \& Lozgka, 2018); and to assess the integrity of the vignette (Bradbury-Jones et al., 2014). In some cases the vignettes were followed up with interviews (Stravakou \& Lozgka, 2018; Wilson \& While, 1998), used for multiple purposes (e.g. discussion, future planning and 
reflection between teachers) (Veal, 2002), or used as one of several data collection tools such as surveys, logs, and interviews to measure the utility of vignettes against other data sources (Stecher et al., 2006).

\section{Design}

The key element of ‘design’ includes practical aspects of vignette construction. Referring to Table 1, the characteristics for this element include: presentation, length, settings and terminology, open or closed questioning, participant perspectives, and piloting.

Presentation. Vignettes vary in the way they are presented and are driven by the aims of the study and data sought. They can be 'static' and portrayed as a 'snapshot' of a situation, or follow a developmental or narrative approach where the vignette unfolds through a series of stages (Jeffries \& Maeder, 2005). Vignettes may be truncated and purposefully left incomplete or abridged where certain details are omitted. It is suggested by Jeffries and Maeder (2005) that truncated vignettes are more open-ended whereas abridged vignettes provide scaffolding as a way of structuring the material within them. Examples of using different types of vignettes appropriate for the aims of the research include: Simon and Tierney (2011) who used open-ended written vignettes both singularly and in parallel; and Stecher et al. (2006) who used two written highly structured vignettes requiring closed-ended responses. Jenkins et al. (2010) specifically compared two different forms of developmental vignettes whereby one was presented as a traditional narrative and the other was made interactive by providing a choice of successive PowerPoint slides.

Visual vignettes might include excerpts from video recordings or other visual representations such as images or cartoons based on 'real-life' events, which offer snapshots of the target stimuli. It is suggested that they can generate rich information, are easier for participants to grasp (Bradbury-Jones, 2014), and can offer reliable data as they are seen to more precisely reflect 'real-life’ situations (Torres, 2010). Friesen and Kuntze (2016, 2018) 
varied the formats in which their vignettes were presented to participants, specifically using text, comic annotation and video formats with identical 'words/dialogue' and mathematical representations to gauge if the format used was influential in the way participants were immersed, appraised, and responded to the vignette formats.

Length. Written vignettes can range from fifty to several hundred words in length. It is suggested that written vignettes remain relatively brief to maintain participants' attention and increase the likelihood of responses (Hughes \& Huby, 2004) and that shorter vignettes may be particularly appropriate for children participants (Bradbury-Jones et al., 2014). Although longer vignettes are likely to add to research demands and time, they can also serve to maintain participants' interest as they develop. In addition, longer unfolding narrative vignettes might prove to be economical if contextual material does not need to be repeatedly provided and can allow for extended details of the vignette material (Hughes \& Huby, 2004). In their unfolding vignettes, Jenkins et al. (2010) used an interactive format by using hyperlinked PowerPoint slides to create plausible scenarios for generating longitudinal data and to enhance the possibility for comparative analysis between different time points.

Although the use of video vignettes was mentioned in several papers, there were no recommendations for how long video stimuli should be. The video clips of classroom teaching vignettes used by Beilstein et al. (2017) lasted less than four minutes. Freisen and Kuntze (2016, 2018) used video vignettes lasting one and a half minutes that could be paused and re-viewed as required.

Settings and terminology. The participants' ability to respond to the vignette is crucial and therefore the setting and language used in the vignette should resonate with potential participants. For example, when seeking the responses of multiple sclerosis patients, Paddam et al. (2010) took care to frame their vignettes to reflect the diversity of the UK population and social environments, and this influenced the choice of characters (e.g., names, 
gender) and expression (e.g., gender-neutral language) used in the vignettes. Using language and names that the participants view as 'typical' is considered important for cultural relevance and community 'norms'. For example, Bradbury-Jones et al. (2014) noted that vignettes should balance familiarity, simplicity/complexity, and the cognitive demands these might place on participants. In some cases the inclusion of technical terminology is a relevant part of the scenario narrative, but this can possibly cause difficulties if participants are unsure of meanings or the content could be perceived differently by participants from diverse backgrounds (Wilson \& While, 1998).

Open or closed questioning. One key advantage of using vignettes is that they provide rich contexts that are conducive for eliciting open-ended responses (Hughes \& Huby, 2004). Vignettes can present complex narratives from which more complicated questions can be asked to elicit detailed responses (Poulou, 2001). Written vignettes often ask for responses in a written form, although the detail and length of expected responses is rarely mentioned.

It is suggested that eliciting responses in a standardized format allows for comparisons to be made more easily, which is useful when analyzing commonalties and differences in participants’ responses. However, using standardized questions in a real-time delivery mode, limits the possibility of asking unscripted questions (Jenkins et al. 2010). In some cases written vignettes might be shown as a stimulus in an interview situation from which participants are asked to respond orally to open-ended questions (Wilson \& White, 1998). In a group situation open-ended questions or unfinished sentences can be used to promote rich discussions (Bradbury-Jones et al., 2014).

Participant perspectives. The perspective from which participants are asked to respond to vignettes can vary depending on the purpose of the research. Perspectives usually include: the vignettes character's viewpoint, a general viewpoint, and the participant’s personal viewpoint (Bradbury-Jones et al., 2014; Hughes \& Huby, 2004). It is suggested that, 
when sensitive topics are being investigated, the vignette narratives are decontextualized to provide distance from 'reality' thus appearing less threatening. This can allow participants to resonate with the vignette characters and to draw on their experiences when responding without feeling uncomfortable. In their study about drug users, Jenkins et al. (2010) described engaging participants in 'Thou-orientations' where they put themselves in the character's place, leading to "valuable insights into their knowledge and experiences of drug treatment" (p. 10). Asking participants to respond from a viewpoint other than their own may produce 'cleaner data' because participants are free to comment on social norms without the fear of making socially undesirable responses (Bradbury-Jones et al., 2014; Hughes \& Huby, 2004; Paddam, 2010) and may also arouse participants’ insights into their own judgment and decision making processes (Torres, 2009). It is also possible to ask participants to respond from more than one perspective such as, for example, to comment about the vignette character and then to imagine themselves in the character's position (Hughes \& Huby, 2004).

Piloting. Although piloting is viewed as a common practice when using vignette methods (Hughes \& Huby, 2004) several of the papers included in this review did not discuss their piloting process (Poulou, 2001; Veal, 2002). Stecher et al. (2006) provided details of developing mathematics classroom scenarios and making "scenario-specific changes on the basis of pilot testing” (p. 109) for their two comparative problem solving scenarios used to elicit elements of teacher practices. Freisen and Kuntze (2016, 2018) asked five experienced teachers who also held positions as teacher educators to evaluate the authenticity of the substance, events, and discourse described in their vignettes as a way of addressing validity. Another way in which credibility of the vignettes can be assessed is when experienced individuals recognize the activity or events portrayed and the data elicited facilitates access to the value-laden constructs sought (Torres, 2009).

\section{Administration}


The final key element of the framework that is outlined in Table 1 concerns the ‘administration' of the vignette and the opportunities for participants to respond. This includes: instructions, timings and responses, and delivery mode and frequency.

Instructions. Specific instructions about procedures to read/view and respond to the vignettes are sometimes omitted or only partially described, whereas in other cases detailed instructions are provided. For example, the participants in Bradbury-Jones et al.’s (2014) study, who were victims of domestic abuse, were informed that the vignette content would be read to them, that it was based on a 'third person', and that they should not draw on their own personal experiences when responding. In contrast, Veal (2002) provided examples and directions for how to use his science and pedagogy vignettes with pre-service teachers in multiple ways. Stecher at al. (2006), who used several written unfolding vignettes depicting hypothetical problems about specific teacher practices, included detailed background information about the characters in their vignettes (such as grade level, student characteristics, topic area, and prior knowledge) and asked participants to respond to the questions as if they were in the situation described.

Timing and responses. In some cases vignettes were given during interviews where participants could read them to identify aspects that viewed as relevant and provide their opinion (Wilson \& While, 1998). Similarly, Stravakou and Lozgka (2018), asked school principals to read each of six vignettes separately, take time to comprehend and then answer questions. In other studies, where the participants were teachers in training, the vignettes were given during their courses (Beilstein et al, 2017; Friesen \& Kuntze, 2018) and Veal (2002) used his vignettes during teacher professional development sessions. Although not many studies provided details about response times, allowing appropriate time for participants to engage meaningfully and thoughtfully with the material to elicit their views is an important aspect to provide for. 
Delivery mode and frequency. Where participants are given vignettes in paper mode they can refer back to them when responding to questions (Stecher et al., 2006). Similarly, some studies that used electronic delivery, such as videos, allowed for repeated viewing (Beilstein, Perry, and Bates, 2017). Friesen and Kuntze (2016; 2018) used three vignette forms (text, comic, and video) to explore pre-service teachers’ level of competency for analyzing hypothetical teacher responses to mathematics problems. They suggested that different vignette modes can affect positioning and contextualization of information for the participants. For example, textual vignettes can provide low levels of individuality that might hinder immersion in the situation; video-based vignettes can provide concrete examples that are high in authenticity but might be difficult for participants to appraise visually and aurally simultaneously; and comic representations require participants to connect graphical and textual information elements to make sense of situations.

Where visual episodes are used it is suggested that a 'script' of the events accompany the visual stimulus to help with recall and viability of responses (Richman \& Mercer, 2002). It is suggested by Richman and Mercer (2002) that if the literacy levels of participants are unknown, then reading the vignette aloud may serve to capture the participants' attention and increase the opportunity for participants to understand and respond to it (Bradbury-Jones et al., 2014). However, oral questioning by researchers might act to constrain the participants’ responses (Hughes \& Huby, 2004) if this reduces time to contemplate their response. Paddam et al. (2010) noted that, if vignettes are being read aloud by the researcher, then the researcher needs to consider the possibility of potential bias and experimenter effects.

The frequency of vignette use is another aspect for consideration. Hughes and Huby (2004) suggested that repeated use of vignettes may lead to a loss of interest from respondents and also risks a 'carry over' effect where the vignette narratives may lose their distinctiveness and ultimately their effectiveness for the research. In contrast, Veal (2002) 
advocated the value of using vignettes multiple times over an extended period of time so that change can be observed and participants are able to reflect on their practice and conceptions of teaching.

\section{Vignette Framework Exemplar: The Mathematics Engagement Study}

In the previous section we have drawn from a range of research that have used vignettes methods to frame what we perceive to be key elements and supporting characteristics for constructing vignettes so that they align with research aims, maintain methodological consistency, and elicit relevant information from participants about the phenomena being investigated. In this section we illustrate the framework for constructing vignettes in the context of a mathematics education research study we conducted (Skilling \& Stylianides, 2015), hereafter referred to as the mathematics engagement study.

The aim of the mathematics engagement study was to elicit individual teacher valueladen beliefs and understandings of student engagement in mathematics and how these influence their practices. We were interested in seeking teacher beliefs specific to mathematics learning and teaching because those beliefs can shape the instructional choices teachers make. More specifically, we were interested in teacher beliefs about promoting student cognitive engagement in mathematics because encouraging student thinking and interest in mathematics is considered important for student learning and future participation (Skilling et al., 2016).

Accordingly, we developed two vignettes narratives describing a 'test preparation’ activity by two fictitious teachers (A and B) (see Appendix A). The narratives were embedded with particular words and phrases that reflected elements of cognitive engagement including self-regulation strategies and metacognitive processes. The description of the two 
fictitious teachers' test preparation differed by the degree to which they promoted selfregulation strategies and metacognitive processes through their teaching approaches. A series of eight open-ended questions were constructed, asking the participants in the study to respond to specific aspects of the two vignettes narratives. In this way, we were able to elicit the participants' beliefs about the practices of the two fictitious teachers as well as their own beliefs and practices for promoting cognitive engagement in mathematics. Next, we provide some background to the constructs of beliefs and cognitive engagement before we illustrate the framework in the context of the mathematics engagement study.

\section{Beliefs and cognitive engagement}

Beliefs are defined as “psychologically held understandings, premises, or propositions about the world that are thought to be true” (Philipp, 2007, p. 259). Individual teacher beliefs include cognitive and emotional aspects about learning and are likened to 'knowledge structures' and reside in the individual. Teachers' practices are influenced by their beliefs and other factors such as the learning contexts and student needs (Bobis et al., 2012), and so being able to investigate teacher beliefs can provide valuable insights to choices of practice.

In mathematics education, the importance of dynamic and active approaches to instruction for enhancing student understanding is at the core of reform-orientated teaching (Stylianides \& Stylianides, 2014). Quality teaching practices for mathematics also identify the importance of attending to students' valuing, confidence, and affect towards mathematics learning. Researchers in mathematics education have highlighted that motivational factors affect students' cognitive processes and motivations for learning, which in turn influence learning outcomes (Stipek et al., 1998). The psychological perspective emphasizes the individual nature of motivational factors that underpin student engagement within the social and cultural milieu of the classroom. 
Cognitive engagement refers to students' approaches to academic tasks as well as their psychological investment in, and willingness to, master complex concepts (Fredricks, Blumenfeld, \& Paris, 2004). Cognitive engagement is not easy to define because it draws from two different but interconnected perspectives. The first associates cognitive engagement with practices that aim to enhance learning and instruction such as using self-regulation strategies and metacognitive processes (Cleary \& Zimmerman, 2012; Wolters \& Taylor, 2012). The second perspective focuses on the psychological investment in learning and motivation, stressing the goals set and efforts students make to master knowledge and skills (Fredricks et al., 2004).

In mathematics learning settings, aspects of cognitive engagement are not always obvious so the goal orientations, self-regulation strategies, and metacognitive processes of students may not be apparent to teachers. A recent study elicited the engagement observations of experienced mathematics and found that teachers' awareness of factors signaling cognitive engagement were limited (Skilling et al., 2016). Therefore, it was important in this study to gain a better understanding of what teachers believed and understood as cognitive engagement and to examine teachers' purported practices to promote their students' engagement for mathematics learning. We decided to use vignettes to facilitate drawing out the beliefs that individual teachers had and to elicit subtleties that exist between different teacher responses (Torres, 2009).

\section{Conception}

Capturing content. For the purposes of this study, we specifically constructed the two vignette narratives (Appendix A). We drew on frameworks of engagement and selfregulation (described in the previous section) to embed particular words and phrases reflecting self-regulation and metacognitive processes such as goal setting, monitoring, and reflecting on revision preparation. Additionally, we drew on literature about reform- 
orientated and traditional pedagogical approaches to distinguish between the practices of the two fictitious teachers in the vignettes. We also drew on our personal experiences of working with secondary mathematics teachers and their students (Veal, 2002). By drawing on relevant frameworks and literature specific to constructs at the core of the research, we were able to align the material within the vignettes to the overall approach and aims of our research.

Realistic and hypothetical portrayals. The vignettes were written to resonate with classroom activities and instructional approaches teachers would be familiar with. We were mindful that information needed to be concrete enough to approximate reality but also allow the participants to form their own interpretations and to help surface their beliefs (Poulou, 2001; Torres, 2009). The context of the vignettes was an upcoming 'maths topic test'. This is a relatively common event in early secondary mathematics classes, one that would be plausible to the participants and based on an activity with which they would have some experience, and therefore they were expected to be able to express their beliefs (Baghramian \& Carter, 2018).

To create two contrasting situations, the narrative of the two vignettes differed by the degree to which Teachers A and B promoted students' use of self-regulation strategies and metacognitive processes. For example, Teacher A handed students a list of topics for revision and told them to revise at home by looking over their notebooks, whereas Teacher B asked students to self-assess their competency, contribute to a 'class revision list' and develop an individual revision plan. Another example is that Teacher A emphasized performance rather than mastery goals, whereas Teacher B expectations that students would use self-regulation strategies to set goals, monitor and reflect on their revision preparation.

Purpose/function. The vignettes were established as the first phase of a multiphase data collection to provide a concrete situation for eight open-ended questions. For example, participants were asked to identify practices of Teacher A and B that they believed were (not) 
important (questions 3 and 4), approaches used by Teacher and B (question 5), and their identification to one of the teachers portrayed (question 6) in the vignettes. The teacher selfidentification also helped ascertain participants for follow-up interviews, where teacher responses could be further unpacked to gain insights into their beliefs (Poulou, 2001; Torres, 2009), how they perceived engagement, and their beliefs about its importance for promoting it in their classrooms.

Each of the methods in the data collection phases elicited rich data for subsequent analysis, and where participants chose to complete additional phases of data collection (e.g., individual interviews and classroom observation), this acted to triangulate the data and increased the researchers' confidence about the findings (Stecher et al., 2006). Additionally, because the subsequent data collection phases were orientated around the purposefully constructed vignettes and accompanying open-ended questions, this was effective for obtaining 'thick descriptions' about teacher beliefs and practices for student engagement. In this way the depth and breadth of data collected assisted with obtaining credible accounts of the participants beliefs and understandings (Simon \& Tierney, 2011).

\section{Design}

Presentation. The vignettes were presented in a written format and portrayed a 'snapshot' of a classroom test preparation activity (Hughes, 1998). The vignettes provided essential but concise information for participants so that the classroom situation portrayed was plausible yet allowed for interpretation by the participants (Stecher et al., 2006).

Length. We were careful that the two vignettes should be similar in length so they appeared equally valued (Hughes \& Huby, 2004). Although the vignettes were contrasting, we ensured similar details about student and teacher activity were provided so that there were equal opportunities for the participants to refer to and respond to their content. 
Settings and terminology. The setting described in the narrative aimed to reflect a typical early secondary mathematics classroom setting and the language avoided using technical terms (Bradbury-Jones et al., 2014). We used written vignettes so that participants could re-read the material as required and refer to it when completing their responses. Also, to facilitate their responses, we assigned line numbers to each vignette.

Open or closed questioning. Eight open-ended questions were used, allowing the participants the opportunity to reflect on the situations presented in the vignettes. For example, participants were asked to indicate where they agreed or disagreed with the fictitious teachers' actions (questions 3 and 4), articulate if they had used approaches of Teacher A and B (question 5) and list additional practices (question 7). Their responses provide information about the distinctive thinking of teachers with different views and varying mathematics experiences (Torres, 2009). The questions were structured in a particular way to elicit different types of responses as we explain in the next section.

Participant perspective. The participants were asked to respond to the vignettes from different perspectives. The first four questions asked teachers to respond from the viewpoint of the teacher character in the vignette. In this way the participants were free to make comments about the choices and practices of the fictitious teachers (Bradbury-Jones, et al, 2014; Hughes \& Huby, 2004). The remaining four questions asked participants to respond from their own perspective reporting their beliefs and choices of practices and how these were similar to or different from those presented by Teachers A and B. Here, the responses provided insights to the participants' thinking, judgments, and decision making (Torres, 2009), which was important information for addressing the research aims of the study.

Piloting. Experienced educators piloted the two contrasting vignettes constructed for this study. This assisted in checking for their authenticity and comparability so that the participants would relate to the narrative portrayed and be able to respond to the questions 
asked of it (Freisen \& Kuntze, 2016). The instructions for the vignettes were also checked for clarity (Stecher et al., 2006); this was crucial because the researchers would not be present at the time of vignette administration or completion.

\section{Key Element: Administration}

Instructions. An introductory paragraph prior to the vignette narratives provided instructions for reading and responding to the vignettes, and also offered brief information about the 'fictitious' class level (Year 7) and useful context (preparation for a mathematics topic test) to help orientate the activity (Friesen \& Kuntze, 2018; Stecher et al., 2006). The instructions indicated that participants could refer to particular phrases in the vignettes in their responses. These references captured individualized responses and allowed for comparisons (Poulou, 2001), therefore providing a deeper understanding of the similarities and differences of teacher beliefs about engagement and practices that promote it (Torres, 2009).

Timings and responses. It was expected that responding to the eight questions would take about 25 minutes. However, the participants were given three weeks to complete and return their responses, allowing them time to reflect on the vignette material (Hughes \& Huby, 2004) and respecting the fact that participants in the study were inservice teachers with a busy work schedule.

Delivery mode and frequency. Sets of vignettes, accompanying questions sheet, information, and consent forms were packaged individually and sent to a nominated lead at each of the schools participating in the research. A self-sealing pre-paid and addressed envelope was included so that individual participants could complete and anonymously return their responses directly to the researchers. 
To conclude, the mathematics engagement study illustrates how underlying theoretical frameworks (in this case self-regulation, goal theory, and metacognition) and approaches to instruction (traditional and reform-orientated) can be suitably embedded within the purposeful construction of vignettes. Our discussion also demonstrates how vignette questions can be tailored and aligned to specific 'events' or 'processes' described in the vignettes, thereby helping elicit teacher beliefs and understandings that influence their practice about student engagement. Finally, our discussion in this section illustrates the potential of using vignettes with other data collection methods, for adding layers of meaning and/or corroborating data.

\section{Concluding Remarks}

In this paper we discussed the affordances of using vignettes methods for exploring complex issues, especially in relation to using vignettes to seek participants’ views about value-laden constructs such as beliefs and understandings. Our discussion situated vignette methods within qualitative research methodologies and an interpretivist paradigm, where the beliefs and understandings of individuals can be sought in unobtrusive ways (Cohen et al., 2011). As suggested by Richman and Mercer (2000), vignettes cut through complex situations and allow for certain kinds of questions to be asked. We were also mindful that teachers felt they could freely convey their individual views and not that any viewpoint was being imposed on them (Torres, 2009). We also argued in this paper that the quality and credibility of qualitative inquiry is crucial and can be strengthened through establishing methodological awareness and consistency (Corbin \& Strauss, 2008; Yilmaz, 2013). Accordingly, not only should the choice of methods align with the research design and theoretical paradigms but the methods themselves should also be developed to be consistent with the research paradigm. 
In developing the vignette framework, we appraised a range of vignette research and identified important aspects for vignette construction (Bradbury-Jones et al., 2014; Hughes \& Huby, 2004; Jeffries and Maeder, 2005; Poulou, 2009; Stecher et al., 2006; Veal, 2002). This resulted in the proposed vignette framework comprising three core elements: conception, design, and administration. We suggest that adherence to these key elements when using vignettes can help align the research aims and methodology and enhance the credibility of the research. Further, using vignettes as part of a sequence of data collections provides opportunities to explore constructs more deeply, adding layers of understanding to the research phenomena (Torres, 2009).

In our exemplar study, the mathematics engagement study, we endeavoured to illustrate aligning the overarching research paradigms, methodologies and methods with relevant theoretical frameworks that represented the constructs underpinning the research. To achieve this, the research design was purposeful as were our vignette constructions. Further, the vignettes and their accompanying questions were also carefully constructed to draw out information particular to the participants' beliefs and practices to better understand the issues and concerns about students' lack of engagement and interest in mathematics.

One of the potential limitations raised when using vignettes are inconsistencies about participants' descriptions of what they would do at an abstract level and what occurs in reality. However, as noted by Jenkins et al. (2010), participants’ espoused beliefs are representative of their intentions rather than predictions of behaviours and are valuable for providing insights into their interpretations and perceptions. In addition, the use of other data collection methods such as follow up interviews, can provide opportunities for clarifying participants' responses and belief structures, while observations of practice can allow for verification of participants' espoused beliefs. We believe that other potential limitations such as difficulty in the construction of the vignette narrative, asking participants about difficult or 
sensitive material, participants being able to comprehend and respond to the vignette questions, etc., are issues that can be resolved if the proposed vignette framework is used.

Not only does the vignette framework provide a template of key elements and characteristics, but it provides a coherent guide for vignette construction and use which we believe is applicable for educational research investigating a range of value-laden constructs.

\section{References}

Baghramian, Maria and Carter, J. Adam, "Relativism", The Stanford Encyclopedia of Philosophy (Winter 2018 Edition), Edward N. Zalta (ed.), URL = https://plato.stanford.edu/archives/win2018/entries/relativism/

Beilstein, S.O., Perry, M., \& Bates, M.S. (2017), Prompting meaningful analysis from preservice teachers using elementary mathematics video vignettes. Teaching and Teaching Education, 63, pp.285-295

Bobis, J., Higgins, J., Cavanagh, M., \& Roche, A. (2012). Professional knowledge of practicing teachers of mathematics. In B. Perry, T. Lowrie, T. Logan, A. MacDonald \& J. Greenless (Eds.), Research in Mathematics Education in Australasia 2008-2011. Rotterdam: Sense Publishers.

Bradbury-Jones, C., Taylor, J. \& Herber, O.R. (2014), Vignette development and administration: a framework for protecting research participants. International Journal of Social Research Methodology, 17(4), pp.427-440

Cleary, T. J., \& Zimmerman, B. J. (2012). A cyclical self-regulation account of student engagement: Theoretical foundations and applications. In A. Christenson, A. Reschly \& C. Wylie (Eds.), Handbook of research on student engagement (pp.237-257). New York: Springer.

Cohen, L. Manion, L. \& Morrison, K. (2011). Research methods in education ( $7^{\text {th }}$ ed.) London: Routledge.

Corbin, J. \& Strauss, A. (2008). Basics of qualitative research: techniques and procedures for developing grounded theory ( ${ }^{\text {rd }}$ ed.) Thousand Oaks: Sage.

Durksen, T. L., Way, J., Bobis, J., Anderson, J., Skilling, K., \& Martin, A. J. (2017). Motivation and engagement in mathematics: A qualitative framework for teacherstudent interactions. Mathematics Education Research Journal. Online first (25 February). doi: 10.1007/s13394-017-0199-1. Available at http://rdcu.be/pBtI

Fredricks, J. A., Blumenfeld, P. C., \& Paris, A. H. (2004). School engagement: potential of the concept, state of the evidence. Review of Educational Research, 74, 59-109. 
Friesen, M., \& Kuntze. S. (2016) Teacher students analyse texts, comics and video-based classroom vignettes regarding the use of representations - does format matter? In Csíkos, c., Rausch, A., Szitány, J. (Eds.), Proceedings of the $40^{\text {th }}$ Psychology of Mathematics Education conference (Vol. 2, pp. 259-266). Szeged, Hungary: PME.

Friesen, M., \& Kuntze. S. (2018) Competence assessment with representations of practice in text, comic and video format. In: Buchbinder, O., Kuntze, S. (eds), Mathematics Teachers Engaging with Representations of Practice (pp.113-130). ICME-14 Monographs. Springer, Cham.

Goldin, G., Rösken, R., and Törner, G. (2009). Beliefs-no longer a hidden variable in mathematical teaching and learning processes. In J. Maaß \& W. Shlöglmann (Eds.). Beliefs and mathematics education. Rotterdam: Sense Publishers.

Hughes, R., \& Huby, M. (2004), The construction and interpretation of vignettes in social research. Social Work \& Social Sciences Review 11(1) pp.36-51

Jeffries, C. Maeder, D.W. (2004), Using vignettes to build and assess teacher understanding of instructional strategies. The Professional Educator, 27 (1\&2), pp.17-28.

Jenkins, N., Bloor, M., Fischer, j. Berney, L., \& Neale, J. (2010), Putting it in context: the use of vignettes in qualitative interviewing. Qualitative Research, 10 (2), pp.175-198.

Nind, M., \& Pepin, B. (2009), Transformation and regulation in educational research, International Journal of Research \& Method in Education, 32, (2), pp.135-136.

Paddam, A. Barnes, D., Langdon, D. (2010), Constructing vignettes to investigate anger in multiple sclerosis. Nurse Researcher, 17 (2), pp.60-73

Philipp, R.A. (2007). Mathematics teachers’ beliefs and affect. In F.K. Lester Jr. (Ed.), Second handbook of research on mathematics teaching and learning (Vol.1, pp.257-315). Charlotte, NC: Information Age Publishing.

Poulou, M. (2001), The role of vignettes in the research of emotional and behavioural difficulties. Emotional and Behavioural Difficulties, 6 (1), pp.50-62.

Richman, J., \& Mercer, D. (2002), The vignette revisited: evil and the forensic nurse. Nurse Researcher 9 (4) pp.70-82

Simon, M. \& Tierney, R. (2011), Use of vignettes in educational research on sensitive teaching functions as assessment. $24^{\text {th }}$ International Congress for School Effectiveness and Improvement $4^{\text {th }}-7^{\text {th }}$ Jan 2011 pp.1-13, Limassol, Cyprus http://www.icsei.net/icsei2011/Full\%20Papers/0153.pdf

Skilling, K., Bobis, J., Martin, A. J., Anderson, J., \& Way, J. (2016). What secondary teachers think and do about student engagement in mathematics. Mathematics Education Research Journal (Vol 28, No.4, pp. 545-566) 
Skilling, K.G. \& Stylianides, G.J. (2015). Promoting cognitive engagement in secondary mathematics classrooms. In K. Krainer \& N. Vondrová (Eds.), Proceedings of the Ninth Conference of the European Society for Research in Mathematics Education (CERME9, 4-8 February 2015) (pp. 1280-1286). Prague, Czech Republic: Charles University in Prague, Faculty of Education and ERME.

Stecher, B., Le, V-N., Hamilton, L., Ryan, G., Robyn, A., \& Lockwood, J.R. (2006), Using structured classroom vignettes to measure instructional practices in mathematics. Educational Evaluation and Policy Analysis, 28 (2), pp.101-130.

Stipek, D., Salmon, J.M., Givvin, K.B., Kazemi, E., Saxe, G., \& MacGyvers, V.L. (1998). The value (and convergence) of practices suggested by motivation research and promoted by mathematics education reformers. Journal for Research in Mathematics Education, 29(4), 465-488.

Stravakou, P.A., \& Lozgka, E.C.(2018). Vignettes in qualitative educational research: Investigating Greek school principals’ values. The Qualitative Report, 23 (5), 1188-1207.

Stylianides, G.J., \& Stylianides, A.J. (2014) The Role of Instructional Engineering in Reducing the Uncertainties of Ambitious Teaching. Cognition and Instruction, 32(4), 374415.

Torres, S. (2009), Vignette methodology and culture-relevance: lessons learned through a project on successful aging with Iranian Immigrants to Sweden. Journal of Cross-Cultural Gerontology, 24 (1), pp.93-114.

Veal, W.R. (2002), Content specific vignettes as tools for research and teaching. Electronic journal of Science Education, 6 (4), June.

Williams, S.R., \& Leatham, K.R. (2017), Journal quality in mathematics education. Journal for Research in Mathematics Education, 48 (4), pp.369-396.

Wilson, J., \& While, A.E. (1998), Methodological issues surrounding the use of vignettes in qualitative research. Journal of Interprofessional Care, 12 (1), pp.79-86

Wolters, C. A., \& Taylor, D. J. (2012). Self-regulated learning perspective on student engagement. In A. Christenson, A. Reschly \& C. Wylie (Eds.), Handbook of research on student engagement (pp. 635-651). New York: Springer.

Yilmaz, K. (2013). Comparisons of quantitative and qualitative research traditions: epistemological, theoretical and methodological differences. European Journal of Education, 48 (2), pp.311-325.

Zimmerman, B. J. and Schunk, D.H. (2008) Motivation, an essential dimension of selfregulated learning. In Schunk, D.H. and Zimmerman, B.J. (Eds.) Motivation and selfregulated learning: Theory, research and applications (pp. 1-30) Mahwah, NJ: Erlbaum.

\section{Additional docs included separately:}




\section{Table 1.}

Appendix A: Teacher vignettes used in the mathematics engagement study

\section{Teacher Vignettes Context}

Two Year 7 mathematics classes at one school will complete a topic test during second term. The teachers of each class provided students with information a week before the test about ways they could prepare. Below are suggestions by Teacher A and Teacher B. Please read each vignette and respond to the questions at the end. The line numbers for each vignette have been included to help make references to the text.

\section{Teacher A}

1. Teacher A reminded students about the upcoming topic test and handed out

2. a sheet with an outline of the key concepts that would likely be covered in

3. the test. The teacher suggested that the students set aside time for revision

4. and to make sure they practised each concept, by looking over their notebooks

5. as it was important for them to achieve a high grade on the test. The teacher

6. also mentioned that the students should ask questions in class if they were

7. unsure of the steps to solve questions. Alternatively, they could come and see

8. the teacher during break time to clarify any questions before the assessment.

9. In each lesson before the test the teacher set five practice questions in case

10. students had not been revising at home and students who got three or less

11. correct were advised they needed to study more.

\section{Teacher B}

12. Teacher B also reminded students about their upcoming topic test. The

13. students were asked to look through their mathematics notebooks and

14. textbooks during the lesson and recall specific topic concepts that they

15. thought would likely to be included in the test. Based on their class

16. work, the students were then asked to record how competent they felt

17. about each concept. During the lesson, the teacher also asked the

18. students to draw on their self-assessment notes and contribute to the

19. creation of a 'class' revision list, from which examples could be revised

20. during lessons before the test. The teacher also told the class that it

21. was expected that each student would develop individual revision

22. plans. Students would work on their individual plans at home, making

23. time to focus on mastering the concepts they believed they needed to

24. improve on. Throughout the week the teacher checked the revision

25. plans of each student and asked how they felt about their preparations.

\section{Open-ended questions:}

1. What are some key similarities you perceive between the two teacher scenarios?

2. What are some key differences you perceive between the two teacher scenarios?

3. List up to two things that each of the teachers did that you believe were important for supporting the students' preparation for the test.

4. List up to two things that each of the teachers did that you do not believe were important for supporting students' test preparation.

5. Have you used any of the approaches used by Teacher A or B? If so, please list them.

6. Overall, which teacher do you identify with the most and why?

7. Can you name any other approaches that are not mentioned in the scenarios that you use to promote homework, study or revision (either during lessons or outside of the classroom)?

8. Choose one of the approaches that you mentioned in the previous question or any approach 
used by Teacher A or Teacher B that you consider important. Would you use or apply this approach for different groups of students? (e.g., Year levels, set or mixed ability classes, girls or boys). Please explain and provide an example if appropriate. 